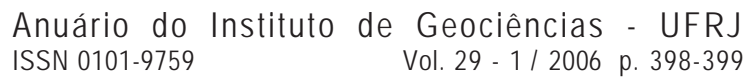

FORAMS 2006

\title{
Modeling of foraminifera: Linking molecules and morphology
}

\author{
Jaroslaw Tyszka ${ }^{1}$ \& Pawe Topa $^{2}$ \\ ${ }^{1}$ Institute of Geological Sciences, Polish Academy of Sciences, \\ Cracow Research Centre, ul. Senacka 1, 31-002 Kraków, Poland \\ ndtyszka@cyf-kr.edu.pl \\ ${ }^{2}$ Institute of Computer Sciences, AGH University of Science and Technology, \\ al. Mickiewicza 30, 30-059 Kraków, Poland
}

Theoretical morphology covers two conceptual areas focused on morphology of organisms, including the simulation of organic morphogenesis and the analysis of the possible spectrum of organic forms via hypothetical morphospace construction. Our studies focus on emergence of shell patterns in the simulated growth of polythalamous foraminifera. Previous models have referred to fixed reference axes and neglected apertures. We present a new approach in foraminiferal modeling applying a moving reference system referred to apertures, which are introduced based on minimization of the local communication path, i.e. a distance between two apertures (Topa \& Tyszka 2002. Lecture Notes in Comp. Sci. 2329: 97-106; Labaj et al., 2003. Lecture Notes in Comp. Sci. 2657: 669-678). The model applies following parameters defining a relative position of a successive chamber: translation factor that controls an overlap of successive chambers, deviation angle (deflection), and rotation angle, as well as the growth factor of chambers applied in the isometric growth. The growth factor can be replaced by 3 additional parameters - scaling ratios - which are defined to simulate allometric chambers. All parameters are either predetermined or selected at random from given ranges that mimic phenotypic variability of a shell.

This moving-reference model focuses on real morphologic characteristics and follows stepwise natural biological processes (Tyszka \& Topa, 2005. Paleobiology, 31 (3): 526-541; Tyszka, 2006. Lethaia, 39 (1): 1-12). The model proves that apertures are essential in foraminiferal morphogenesis. Simulated foraminiferal shells based on this model indicate that their varieties are much wider than morphotypes developed by fixed reference models. It is especially well suited for simulating gradual and abrupt changes in chamber growth patterns as features characteristic for real foraminiferal shells. For 
instance, switchovers from planispiral or streptospiral to biserial or uniserial shell arrangements can be simulated. Nevertheless, this model is still not able to simulate forms with the longest global communication path connecting foramina, such as Lenticulina or other lagenids. This is due to the assumptions that chambers are spheroidal and that an aperture does not shape any chamber. On the other hand, empirical observations have shown that apertures often have a strong impact on the shape of a chamber, e.g., chambers tend to show specific apertural structures, such as necks, radial structures, depressions around apertures etc. In order to simulate such forms, it is necessary to leave this geometric approach and focus on more "in-depth" models based on realistic intracellular dynamics (Tyszka \& Topa, 2005; Tyszka, 2006; Tyszka et al., 2005. Studia Geol. Polon., 124: 143-157).

This novel approach has been tested based on the Diffusion-Limited Aggregation (DLA) model that mimics formation of the foraminiferal cytoskeleton (Tyszka et al., 2005). The simulated two-dimensional forms resemble either a reticulopodium or a microtubular fan supporting the organic 'Anlage' during the chamber formation. The results are promising, but the model still needs further development. We suppose that the emergent model is the best alternative for foraminiferal modeling. Such a future model should incorporate recent knowledge on cytoskeletal dynamics focused on simplified interactions of proteins.

This research is sponsored by the Polish Ministry of Education and Science (Grant nr 3 PO4D 048 24). For more details see: http:// www.eforams.icsr.agh.edu.pl/ 